\title{
Extreme Movements of the Major Currencies traded in Australia
}

\author{
Chow-Siing Sia ${ }^{a}$, Felix Chan ${ }^{\text {a }}$ \\ ${ }^{a}$ School of Economics and Finance, Curtin University \\ Email: chow.sia@cbs.curtin.edu.au
}

\begin{abstract}
Following the event such as Global Financial Crisis (GFC) in 2008, financial institutions have suffered significant financial losses. Central banks have continuously bailed out financial distressed firms. However, such decisions from central banks cast doubt on the adequacy of current risk management strategy to reduce risk. Hence, the necessity of establishing robust risk evaluation techniques to manage losses during extreme events is critical. In particular, Value-at-Risk (VaR) has become an important measure of risk since the implementation of Basel II in 2008 and continues to become an important feature in Basel III. Even though the properties of $\mathrm{VaR}$ are well established, robust construction and forecast of VaR based on historical data remain unresolved. This is due to the difficulties in identifying the dynamics in asset's returns and it is extremely difficult to construct robust forecast without knowing the underlying process that governs the data. This paper proposes to estimate VaR by applying the extended version of the Hill's (1975) tail index estimator proposed in Berkes, et al. (2003). The extension incorporates potential time varying conditional variance and thus, provides a more robust method to estimate the tail index.

The paper will provide a concise overview on various tail index estimators and their extensions, including Mikosch and Starica (2000), Berkes, et al. (2003), Iglesias and Linton (2009) and Hill (2010). It also contains a brief overview on some of the popular conditional variance models, including the Generalized Autoregressive Conditional Heteroskedasticity (GARCH) model of Bollerslev (1986) and its asymmetric extension by Glosten, Jagannathan and Runkle (1993).

This paper will then forecast the VaR of exchange rate returns by estimating the tail index using daily exchange rate data on AUD with USD and GBP from January 1984 to December 2012. This approach is based on Iglesias and Linton (2009), Iglesias (2012) and Iglesias and Lagoa Varela (2012), which applied the tail index estimator proposed in Mikosch and Starica (2000) and Berkes, et al. (2003). The paper will also present a comparison of VaR between different conditional variance models suggested by Jorion (1996, 2007) to investigate the robustness of VaR estimates by tail index.
\end{abstract}

Keywords: GARCH-type estimators, tail index, Value-at-Risk, exchange rates 


\section{INTRODUCTION}

In recent years, extreme events, such as the Global Financial Crisis (GFC), have caused significant losses in financial institutions, central banks have continuously bailed out financial distressed firms. Such decisions from central banks cast doubt on the adequacy of current risk management strategy to reduce risk. Hence, the necessity of establishing robust risk evaluation techniques to manage losses during extreme events is critical. In particular, Value-at-Risk (VaR) has become an important measure of risk since the introduction of Basel II in 2008. It is defined to be the probability of worst cumulative loss on a bank trading outcomes over a holding period within a given level of confident (Jorion, 1996, 2007). While the debate on the effectiveness of $\mathrm{VaR}$ as a risk measure remains controversial, VaR continues to be an important feature in Basel II and III. Even though the properties of VaR are well established, robust construction and forecast of VaR based on historical data remain unresolved. This is due to the difficulties in identifying the dynamics in asset's returns and it is extremely difficult to construct robust forecast without knowing the underlying process that governs the data. For this reason, VaR has attracted considerable attention in the literature, which focuses on statistical descriptions and assessment of different modeling techniques and approaches. A study by Berkowitz and O'Brien (2002) question the accuracy of VaR models on large trading portfolios at commercial banks by presenting structural complication in VaR forecasts estimated by banks in measuring risk.

On the other hand, Hill (1975), Berkes, et al. (2003) and Hill (2010) propose different estimators for tail index under different assumptions of the data generating processes. The seminal work in Hill (1975) proposes an estimator of tail index based on a sample of independently and identically distributed random variables. Mikosch and Starica (2000) and Berkes, et al. (2003) generalise the Hill's (1975) estimator by assuming the data generating process follows a $\operatorname{GARCH}(1,1)$ model. Hill (2010) specifies the estimator further to accommodate a much wider class of data generating processes. Recent studies by Iglesias (2012) and Iglesias and Lagoa Varela (2012) apply those estimators for tail index under the assumption that the conditional variances of stock and exchange rate returns follow a $\operatorname{GARCH}(1,1)$ process. Extreme exchange rate movements can be described as the lowest and the highest changes in exchange rate returns over a period of time (Aggarwal and Min, 2009). From a probabilistic viewpoint point, extreme observation belongs to the tail of the distribution. Therefore a tail index contains valuable information, which reflects the behavior of exchange rate movements (Hols and De Vries, 1991).

Empirical studies on extreme movements using tail index in foreign exchange markets are numerous. A notable study by Wagner and Marsh (2005) perform a simulation analysis of tail index on exchange rate returns to allow for small sample bias in determining the tail thickness of extreme exchange rate movements. While, Payaslioğlu (2009) examines the extent to which extreme movements of exchange rate returns can be measured to test for structural changes due to unexpected shifts following the development of different exchange rate regimes in Turkey. Nevertheless, Cotter (2005) concentrates on the tail index of Euro currencies movements to explore the possibility of speculative pressure prior European Monetary System (EMS) crisis in 1992. More importantly, the relationship between tail index and VaR of the unconditional distribution is explored in the studies of Iglesias and Linton (2009), Iglesias (2012) and Iglesias and Lagoa Varela (2012). As a result, the tail index provides an alternative avenue to estimate VaR.

This paper forecasts the $\mathrm{VaR}$ of exchange rate returns by estimating the tail index using daily exchange rate data on USD/AUD and GBP/AUD. This approach is based on Iglesias and Linton (2009), Iglesias (2012) and Iglesias and Lagoa Varela (2012), which applied the tail index estimator proposed in Mikosch and Starica (2000) and Berkes, et al. (2003). The paper will also present a comparison of VaR between different conditional variance models suggested by Jorion $(1996,2007)$. Hence, the robustness of VaR estimates by tail index can also be investigated.

This paper is organized as follows: Section 2 explains the model, and a selection of adaptive methods for Hill estimation to test the model. Data and main results are presented in Section 3. Finally, a conclusion is drawn in Section 4.

\section{MODEL AND ESTIMATOR FOR TAIL INDEX}

This section provides a concise overview on the estimator for tail index under the Generalized Autoregressive Conditional Heteroskedasticity (GARCH) model of Bollerslev (1986) and its asymmetric extension (GJR) by Glosten et al. (1993). 
Let $r_{t}$ denotes the exchange rate return at time $t$ such that:

$$
r_{t}=\log \left(\frac{s_{t}}{s_{t-1}}\right)
$$

where, $s_{t}$ denotes the exchange rate of an Australian dollar to a foreign currency at time $t$ for $t=2, \ldots, \mathrm{T}$.

Consider the GARCH(1,1) model of Bollerslev (1986)

$$
\begin{aligned}
& r_{t}=\sigma_{t} \eta_{t} \quad \eta_{t} \sim \operatorname{iid}(0,1) \\
& \sigma_{t}^{2}=\omega+\alpha r_{t-1}^{2}+\beta \sigma_{t-1}^{2}
\end{aligned}
$$

with $\omega \geq 0, \alpha>0$, and $\beta \geq 0$. The parameters of the model can be estimated by Maximum Likelihood Estimator (MLE) under normality, which becomes Quasi-MLE (QMLE) if $\eta_{t}$ does not follow a normal distribution. Bougerol and Picard (1992) showed that if the log-moment condition,

$$
E \log \left(\alpha \eta_{t}^{2}+\beta\right)<0
$$

is satisfied, then $\operatorname{GARCH}(1,1)$ is stationary and ergodic. Moreover, under the same condition, Jeantheau (1998) and Boussama (2000) showed that QMLE is consistent and asymptotically normal, respectively. Ling and McAleer (2003) provided necessary and sufficient conditions for stationarity and ergodicity as well as sufficient conditions for consistency and asymptotic normality of $\operatorname{QMLE}$ for $\operatorname{GARCH}(p, q)$ but their results require slightly stricter assumptions than the log-moment condition.

Glosten et al. (1993) proposed an alternative specification to the conditional variance equation aiming to capture the asymmetric effects of shocks on the conditional variance. The GJR(1,1) model can be written as:

$$
\sigma_{t}^{2}=\omega+\left(\alpha+\gamma I_{t-1}\right) r_{t-1}^{2}+\beta \sigma_{t-1}^{2}
$$

where

$$
I_{t}=\left\{\begin{array}{cc}
0 & r_{t}>0 \\
1 & r_{t} \leq 0
\end{array}\right.
$$

McAleer, et al. (2007) showed that the log-moment condition for GJR(1,1) model, namely

$$
E \log \left[\left(\alpha+\frac{1}{2} \gamma\right) \eta_{t}^{2}+\beta\right]<0
$$

is sufficient for consistency and asymptotic normality of QMLE.

Note that by Jensen's inequality, $\log E\left(\alpha \eta_{t}^{2}+\beta\right)<E \log \left(\alpha \eta_{t}^{2}+\beta\right)<0 \Rightarrow E\left(\alpha \eta_{t}^{2}+\beta\right)-1<0$. Similar observation holds for the log-moment condition of GJR by simply replacing $\alpha$ by $\alpha+0.5 \gamma$ in the previous expression. Let $\hat{\alpha}, \hat{\beta}, \hat{\eta}_{t}$ be consistent estimates of $\alpha, \beta, \eta_{t}$, respectively. Define $A_{t}=\alpha \eta_{t}^{2}+\beta$ such that $\hat{A}_{t}=\hat{\alpha} \hat{\eta}_{t}^{2}+\hat{\beta}$, then Mikosch and Starica (2000) and Berkes, et al. (2003), proposed to estimate the tail index, $\kappa$, by solving:

$$
\hat{\phi}_{T}(\kappa)=0
$$

where

$$
\hat{\phi}_{T}(\kappa)=T^{-1} \sum_{t=1}^{T} \hat{A}_{t}^{\frac{\kappa}{2}}-1 .
$$

When $\kappa=2$, equation (4) can be viewed as the sample estimate of the log-moment condition. Moreover, if equation (3) is true when $\kappa=2$, it implies that the log-moment condition does not hold. Thus, equation (6) provides an alternative way to test the validity of the log-moment condition given the fact that $\hat{\kappa}$ is consistent and asymptotically normal as shown in Berkes et al. (2003). Similar results can be obtained for GJR(1,1) 
model by replacing $\alpha$ with $\alpha+0.5 \gamma$ in the definition of $A_{t}$ and $\hat{\phi}_{T}(\kappa)$ as shown in Iglesias and Linton (2009).

Iglesias and Linton (2009) also demonstrated the relationship between $\kappa$ and VaR for a given significant level, $v$. Formally,

$$
v=\operatorname{Pr}\left[r_{t}>\operatorname{VaR}{ }_{v}\right] \equiv c_{0} \operatorname{VaR} R_{v}^{-\kappa}
$$

which implies

$$
\operatorname{VaR}_{v}=\left(\frac{c_{0}}{v}\right)^{\frac{1}{\kappa}} .
$$

Furthermore, Iglesias and Linton (2009) showed that

$$
\hat{c}=\frac{1}{2 T} \sum_{t=1}^{T}\left|\hat{\eta}_{t}\right|^{\hat{\kappa}} \frac{\sum_{t=1}^{T}\left[\left(\hat{\omega}+\hat{A}_{t} \hat{\sigma}_{t}^{2}\right)^{\hat{\kappa} / 2}-\left(\hat{A}_{t} \hat{\sigma}_{t}^{2}\right)^{\hat{\kappa} / 2}\right]}{\frac{\hat{\kappa}}{2} \sum_{t=1}^{T} \hat{A}_{t}^{\hat{\kappa} / 2} \ln \hat{A}_{t}}
$$

is a consistent estimator for $c_{0}$. Therefore $\mathrm{VaR}$ can be estimated as

$$
\widehat{\operatorname{VaR}}_{v}=\left(\frac{\hat{c}}{v}\right)^{\frac{1}{\hat{k}}}
$$

Equations (9) and (10) apply to both $\operatorname{GARCH}(1,1)$ and $\operatorname{GJR}(1,1)$ models with the appropriate definitions of $A_{t}$ and $\sigma_{t}^{2}$. Suggested by Jorion $(1996,2007)$, VaR can be measured under two conditions. If exchange rate movements are assumed to be normally distributed, conditional $\mathrm{VaR}$ can be derived directly from the mean and standard deviation of exchange rate returns. In which case, $V a R_{t}$ is estimated at 99 percent confidence level. Hence,

$$
\operatorname{VaR}_{t}=\hat{r}_{t}-t_{\alpha / 2} \hat{\sigma}_{t}
$$

where, $\hat{r}_{t}$ is the forecasted exchange rate return at time $t ; t_{\alpha / 2}$ is the critical value from the standardized distribution of $\hat{r}_{t}$ at a value of 2.327, and $\hat{\sigma}_{t}$ is the estimated conditional standard deviation of $\hat{r}_{t}$. Alternatively, a sigma-based $V a R_{t}$ of exchange rate movements can be measured to accommodate for the extreme movements of exchange rate returns, particularly when the distribution of exchange rate returns unlikely to be normally distributed and the standardized residuals appear to be fat-tailed ${ }^{1}$. Following equation (12), $\hat{\sigma}_{t}$ is replaced with the estimated standard deviation of $\hat{r}_{t}$ from $\operatorname{GARCH}(1,1)$ model in equation (2).

\section{RESULTS}

\subsection{Data}

Daily exchange rates on USD/AUD and GBP/AUD are collected from Thomson Reuters DataStream Professional, from the period of 1 January 1984 to 31 December 2012. These periods are chosen in order to capture as many major financial events as possible. This includes US stock market crash in 1987, EMS crisis in 1992, Asian currency crisis in 1997 and GFC in 2008.

\subsection{Main Results}

A summary statistics of the exchange rate returns is presented in Table 1. USD/AUD provides a maximum value of 0.07156 and a minimum value of -0.07737 with 0.0072667 standard deviation. While, GBP/AUD shows a maximum value of 0.05707 and a minimum value of -0.06695 with 0.0075052 standard deviation. USD/AUD has a higher mean value compared to GBP/AUD. Both USD/AUD and GBP/AUD are negatively skewed at a value of -0.42234 and -0.16358 , respectively, displaying greater value of kurtosis for USD/AUD at 11.05533, while GBP/AUD at 6.90985.

\footnotetext{
${ }^{1}$ See Hols and De Vries (1991), Wagner and Marsh (2005)
} 
Table 1. Summary statistics of USD/AUD and GBP/AUD returns

\begin{tabular}{lcc}
\hline & $\begin{array}{c}\text { USD/AUD } \\
(\mathrm{n}=7565)\end{array}$ & $\begin{array}{c}\text { GBP/AUD } \\
(\mathrm{n}=7565)\end{array}$ \\
\hline Mean & 0.00001861 & 0.00000467 \\
Standard Deviation & 0.007266711 & 0.007505233 \\
Minimum & -0.07737 & -0.06695 \\
Maximum & 0.07156 & 0.05707 \\
Skewness & -0.42234 & -0.16358 \\
Kurtosis & 11.05533 & 6.90985 \\
\hline
\end{tabular}

The time series of the daily exchange rate returns of USD/AUD and GBP/AUD are illustrated in Figure 1 and Figure 2. Figure 3 and Figure 4 present the histogram of the time series data. A strong magnitude of exchange rate fluctuations can be observed particularly in the periods of 1987 and 2008 due to US stock market crashes and GFC in both USD/AUD and GBP/AUD exchange rate returns. Similarly, GBP/AUD exhibits a strong movement in the event of EMS crisis during the periods of 1992 and 1993.

The daily volatilities of $\mathrm{VaR}_{t}$ for AUD/USD and GBP/AUD are also plotted in Figure 1 and Figure 2 beneath the daily exchange rate returns. It can be observed that the time-varying characteristics of the conditional volatilities between the exchange rate returns are evident with periods of high and low volatilities.

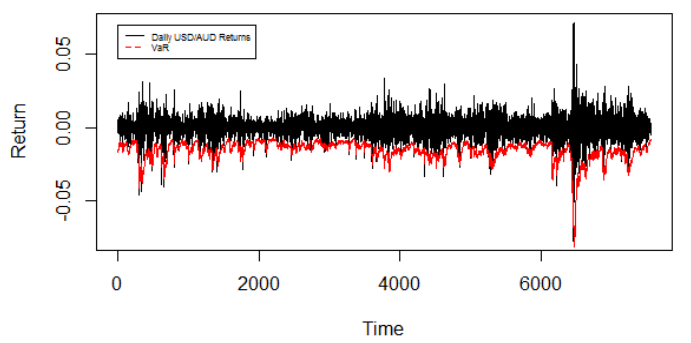

Figure 1. Daily USD/AUD Returns

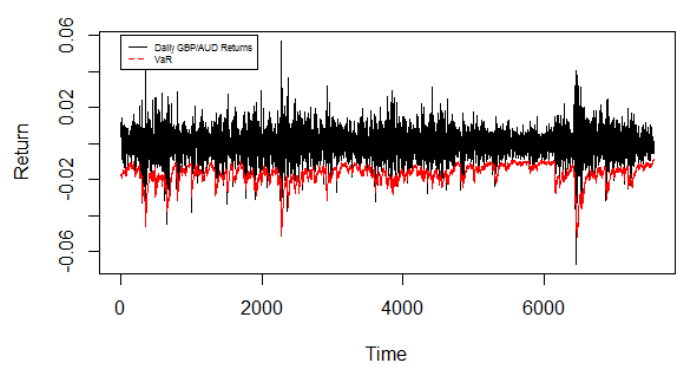

Figure 2. Daily GBP/AUD Returns

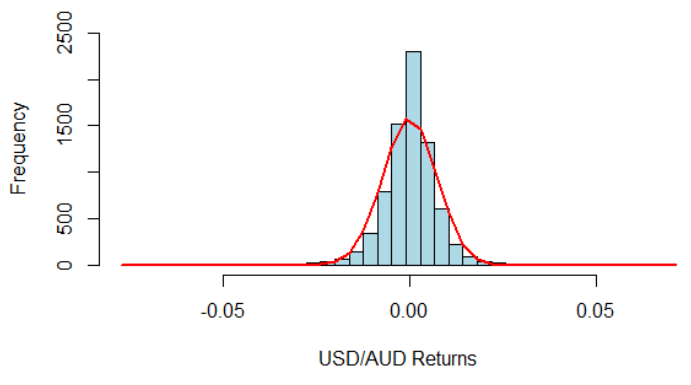

Figure 3. Histogram of USD/AUD Returns

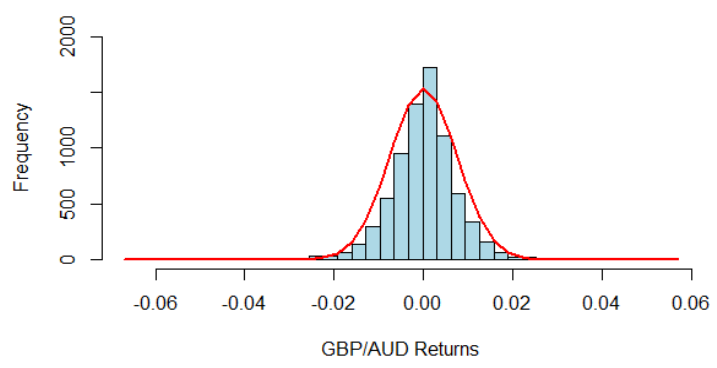

Figure 4. Histogram of USD/AUD Returns

Table 2 presents the estimates for $\widehat{\omega}, \widehat{\alpha}, \hat{\beta}$, and $\hat{\gamma}$ in $\operatorname{GARCH}(1,1)$ and $\operatorname{GJR}(1,1)$ models for daily exchange rate returns of USD/AUD and GBP/AUD. For $\operatorname{GARCH}(1,1)$, the estimates for $\widehat{\omega}, \widehat{\alpha}, \hat{\beta}$ are positive for both exchange rate returns, where $\sigma_{t}^{2}>0$. Notice that $\widehat{\alpha}+\hat{\beta}$ is very close to 0.99 , which implies that the shocks to the conditional variance is persistent.

In $\operatorname{GJR}(1,1)$ model, the estimates for $\widehat{\omega}, \widehat{\alpha}, \hat{\beta}$ and $\hat{\gamma}$ are also positive for both exchange rate returns, where $\sigma_{t}^{2}>0$. One can observe in the case of USD/AUD, $\hat{\beta}$ estimate from $\operatorname{GJR}(1,1)$ model has decreased. However, in the case of GBP/AUD, $\hat{\beta}$ estimate from $\operatorname{GJR}(1,1)$ model has increased. While, $\widehat{\omega}$ and $\widehat{\alpha}$ 
estimates remain very small. At the same time, $\hat{\gamma}$ estimates in GJR(1,1) model for USD/AUD and GBP/AUD are represented at 0.02350477 and 0.03198424 , respectively.

It is also interesting to note that t-values for both exchange rate returns are significant for each of the parameters in $\operatorname{GARCH}(1,1)$ and GJR(1,1) models. Likewise, the standard errors are also significant except $\widehat{\omega}$ estimates with zero values.

Table 2. Parametric estimation

\begin{tabular}{ccccccc}
\hline GARCH(1,1) & USD/AUD & Standard Error & $t$-value & GBP/AUD & Standard Error & $t$-value \\
\hline$\omega$ & $4.94 \mathrm{e}^{-07}$ & 0 & 6.1607 & $5.93 \mathrm{e}^{-07}$ & 0 & 4.9223 \\
$\alpha$ & 0.07049178 & 0.005273 & 13.4195 & 0.06289407 & 0.005134 & 12.2514 \\
$\beta$ & 0.921479 & 0.005446 & 169.1933 & 0.9278593 & 0.006 & 154.6491 \\
\hline GJR $(1,1)$ & & & & & \\
\hline$\omega$ & $5.32 \mathrm{e}^{07}$ & 0 & 6.32935 & $5.93 \mathrm{e}^{-07}$ & 0 & 5.080991 \\
$\alpha$ & 0.05728914 & 0.006355 & 9.02631 & 0.04236209 & 0.00579 & 7.309587 \\
$\beta$ & 0.92123 & 0.005632 & 163.55118 & 0.9315636 & 0.005913 & 157.5502 \\
$\gamma$ & 0.02350477 & 0.007795 & 3.01139 & 0.03198424 & 0.00693 & 4.601914 \\
\hline
\end{tabular}

Table 3. Estimated values of $\hat{\kappa}, \hat{c}$ and $\widehat{V a R}_{v}$, sigma-based $V a R_{t}$ and conditional $V a R_{t}$, where $\alpha=0.01$

\begin{tabular}{clll}
\hline GARCH(1,1) & & USD/AUD & GBP/AUD \\
$\hat{k}$ & Mikosch and Starica (2000) & 3.675781 & 4.548828 \\
$\hat{c}$ & Berkes et al. (2003) & $1.06 \mathrm{e}^{-08}$ & $4.97 \mathrm{e}^{-10}$ \\
$\widehat{\operatorname{VaR}}_{v}$ in (11) & Iglesias and Linton (2009) & 0.02367349 & 0.02479983 \\
\hline Sigma-based $\mathrm{VaR}_{t}$ in (12) & Jorion (1996, 2007) & 0.01571949 & 0.01678839 \\
\hline Conditional $\mathrm{VaR}_{t}$ in (12) & Jorion (1996, 2007) & 0.01688628 & 0.01745512 \\
\hline GJR (1,1) & & USD/AUD & GBP/AUD \\
$\hat{k}$ & Mikosch and Starica (2000) & 4.042358 & 5.124023 \\
$\hat{c}$ & Berkes et al. (2003) & $2.56 \mathrm{e}^{-09}$ & $6.13 \mathrm{e}^{-11}$ \\
$\widehat{\operatorname{VaR}}_{v}$ in $(11)$ & Iglesias and Linton (2009) & 0.02339781 & 0.02496014 \\
\hline
\end{tabular}

Table 3 shows all results of $\hat{\kappa}, \hat{c}, \widehat{V a R}_{v}$ following $\operatorname{GARCH}(1,1)$ and $\operatorname{GJR}(1,1)$ models, as well as sigmabased $\operatorname{VaR}_{t}$ and conditional $\mathrm{VaR}_{t}$, following Equation (12).

It can be observed that $\hat{\kappa}$ values for both exchange rate returns in $\mathrm{GJR}(1,1)$ model present greater values. While, $\hat{c}$ values for both exchange rate returns in $\operatorname{GJR}(1,1)$ model present lower values. At the same time, $\widehat{V a R}_{v}$, following Equation 11, for both exchange rate returns are equitably constant at values of 0.024. One can also note that conditional $V_{a}$ for both exchange rate returns are at greater values than sigma-based $V a R_{t}$. Nevertheless, conditional and sigma-based $V a R_{t}$ for both exchange rate returns appear to be less significant than $\widehat{\operatorname{VaR}}_{v}$.

\section{CONCLUSIONS}

In this paper, both conditional and unconditional variance of exchange rate returns between daily exchange rate returns of USD/AUD and GBP/AUD are investigated. A comparison between GARCH $(1,1)$ and GJR $(1,1)$ models is established to provide additional evidence on the distribution of exchange rate returns under extreme conditions. The estimation of tail index presents a good indicator which is estimated by means of robust techniques by Mikosch and Starica (2000), Berkes et al. (2003), and Iglesias and Linton (2009), to provide more information than reporting conditional variance on daily exchange rate returns only. It is also interesting to note that conditional and sigma-based $V a R_{t}$ for both exchange rate returns appear to be less significant than $\widehat{\operatorname{VaR}}_{v}$. 
Chow-Siing Sia, Felix Chan, Extreme movements of the major currencies traded in Australia

\section{ACKNOWLEDGEMENT}

The authors would like to thank the two anonymous referees and the financial support from the Australian Research Council is also gratefully acknowledged.

\section{REFERENCES}

Aggarwal, R., and Q. Min. (2009). Distribution of extreme changes in asian currencies: Tail index estimates and value-at-risk calculations. Applied Financial Economics, 19(13), 1083-1102.

Berkes, I., Lajos H., and P. Kokoszka. (2003). Estimation of the maximal moment exponent of a GARCH(1,1) sequence. Econometric Theory, 19(4), 565-586.

Berkowitz, J., and James O'Brien. (2002). How accurate are value-at-risk models at commercial banks? Journal of Finance, 57(3), 1093-1111.

Bollerslev, T. (1986). Generalized autoregressive conditional heteroscedasticity. Journal of Econometrics, 31, 307-327.

Bougerol, P. and N. Picard. (1992). Stationarity of GARCH processes and of some non-negative time series. Journal of Econometrics, 52, 115-127.

Boussama, F. (2000). Asymptotic normality for the quasi-maximum likelihood estimator of a GARCH model. Comptes Rendus de l'Aacdemie des Sciences, Serie I 331, 81-84 (in French).

Cotter, J. (2005). Tail behaviour of the euro. Applied Economics, 37(7), 827-840.

Glosten, L., R. Jagannathan, and D.E. Runkle. (1993). On the relation between the expected value and the volatility of the nominal excess return on stocks. Journal of Finance, 48(5), 1779-1801.

Hill, B.M. (1975). A simple general approach to inference about the tail of a distribution. The Annals of Statistics, 3(5), 1163-1174.

Hill, J.B. (2010). On tail index estimation for dependent, heterogeneous data. Econometric Theory, 26(05), 1398-1436.

Hols, M.C.A.B., and C.G. De Vries. (1991). The limiting distribution of extremal exchange rate returns. Journal of Applied Econometrics, 6(3), 287-302.

Iglesias, E.M. (2012). An analysis of extreme movements of exchange rates of the main currencies traded in the foreign exchange market. Applied Economics, 44 (35), 4631-4637.

Iglesias, E.M., and M.D. Lagoa Varela. (2012). Extreme movements of the main stocks traded in the eurozone: An analysis by sectors in the 2000's decade. Applied Financial Economics, 22 (24), 2085-2100.

Iglesias, E.M. and B.O. Linton. (2009). Estimation of tail thickness parameters from GARCH models. Working Paper, Michigan State University and Universidad Carlos III de Madrid.

Jeantheau, T. (1998). Strong consistency of estimators for multivariate ARCH models. Econometric Theory, $14,70-86$.

Jorion, P. (1996). Risk 2: Measuring the risk in value at risk. Financial Analysts Journal, 52 (6), 47-56.

Jorion, P. (2007). Value at Risk: The New Benchmark for Managing Financial Risk, 3rd ed. The McGrawHill Companies Inc., New York.

Ling, S. and M. McAleer. (2003). Asymptotic theory for a vector ARMA-GARCH model. Econometric Theory, 19, 278-308.

McAleer, M., F. Chan and D. Marinova. (2007). An econometric analysis of asymmetric volatility: Theory and application to patents. Journal of Econometrics, 139, 259-284.

Mikosch, T., and C. Starica. (2000). Limit theory for the sample autocorrelations and extremes of a GARCH $(1,1)$ process. The Annals of Statistics, 28(5), 1427-1451.

Payaslioğlu, C. (2009). A tail index tour across foreign exchange rate regimes in turkey. Applied Economics, 41(3), 381-397.

Wagner, N., and T.A. Marsh. (2005). Measuring tail thickness under GARCH and an application to extreme exchange rate changes. Journal of Empirical Finance, 12(1), 165-185. 